\title{
Quantitative oscillator analysis of IR-optical spectra on spin-cast chitosan films
}

\author{
W.H. Nosal ${ }^{\mathrm{a}}$, D.W. Thompson ${ }^{\mathrm{a}}$, S. Sarkar ${ }^{\mathrm{b}}$, A. Subramanian ${ }^{\mathrm{b}}$ and J.A. Woollam ${ }^{\mathrm{a}, *}$ \\ ${ }^{a}$ Department of Electrical Engineering, University of Nebraska, Lincoln, NE 68588, USA \\ ${ }^{\mathrm{b}}$ Department of Chemical Engineering, University of Nebraska, Lincoln, NE 68588, USA
}

\begin{abstract}
Infrared optical properties of spin-cast chitosan films have been determined using spectroscopic ellipsometry. Infrared index of refraction and extinction coefficients from $750 \mathrm{~cm}^{-1}$ to $4000 \mathrm{~cm}^{-1}$ were determined using ellipsometric data fits to dispersion models based on Gaussian shaped oscillators. The free electron contribution was analyzed using a Drude model. This modeling determined that optical anisotropy was present over the entire infrared region. Line shape and oscillator strength analysis was performed to determine oscillator strengths, abundance, and relative bond strength.
\end{abstract}

Keywords: Bond occurrence, chitosan, ellipsometry, infrared spectrum, oscillator strength

\section{Introduction}

Biopolymers, such as cellulose, starch, glycogen and chitin, compose a significant portion of the biomass on the planet. Chitin is a polymeric carbohydrate and a major component in shells of crustaceans such as shrimp and crabs [1]. Chitin consists of repeated units of N-acetyl-D-glucosamine joined together by $\boldsymbol{\beta}(1 \rightarrow 4)$ linkages. Chitosan is a chemically modified form of chitin where the acetyl group attached to the nitrogen atom is replaced by hydrogen through hydrolysis, yielding a primary amine group. Both chitin and chitosan have a chemical structure similar in nature to cellulose. The structures of chitin, chitosan, and cellulose are shown in Fig. 1.

\section{Experimental procedure}

\subsection{Sample preparation}

For this study, thin films of chitosan were produced by spin casting a solution of chitosan onto $\left(\begin{array}{lll}1 & 0 & 0\end{array}\right)$ orientated silicon wafers (manufactured by Polishing Corporation of America). Wafers were 2 inches in diameter, $0.3-0.4 \mathrm{~mm}$ thick, polished on one side and typically had $1.6 \mathrm{~nm}$ of native oxide [2].

Solutions used to produce films were $2 \%(\mathrm{w} / \mathrm{v})$ of chitosan to solvent. The solvent was a $2 \%(\mathrm{w} / \mathrm{v})$ aqueous acetic acid solution with a pH of 2.6. Chitosan is soluble in acidic conditions. Powdered chitosan and acetic acid solution were mixed at room temperature on a stir plate for 18 hours yielding a viscous liquid with a faint brown color. Viscosities of about 825 centipoise (cP) were found, as measured on a commercial rotating cylinder viscometer.

\footnotetext{
${ }^{*}$ Corresponding author. Tel.: +1 402477 7501, ext. 227; Fax: +1 402477 8214; E-mail: jwoollam@jawoollam.com.
} 

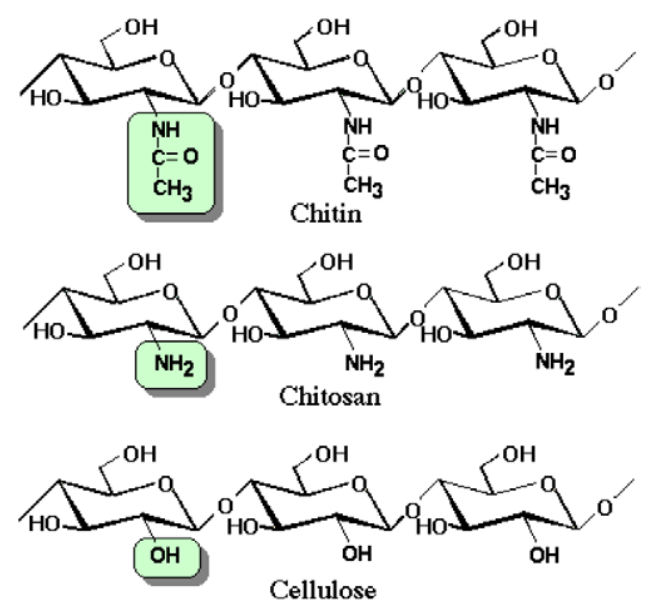

Fig. 1. Structures of chitin and chitosan and their comparison to cellulose.

The chitosan solutions initially yielded films having numerous small spot and comet defects, due to small undissolved impurity particles. These particles caused spot defects through capillary action, creating areas of greater film thickness around the particle. Because of high solution viscosity, small particle size, and possibly a small difference in particle to solution density, an ultracentrifuge was used for 5 hours with $27,500 \mathrm{~g}$ acceleration to remove particulates from suspension, this yielded films free of defects $[2,3]$.

Silicon wafers were cleaned by washing with acetone, then ethanol, followed by de-ionized water. The wafer was air dried using pressurized dry air supply. Five milliliters of chitosan solution were dispensed onto the center of the wafer and allowed to flow to the edges, the acceleration was ramped at its slowest setting to a maximum spin speed of 2500 RPM and allowed to spin for 30 seconds. By pre-loading the solution and using the lowest acceleration ramp, comets and other typical spin coat defects were eliminated or greatly reduced.

After spinning, the sample was removed and allowed to air dry. Acidity of the chitosan film was neutralized by immersion in $0.25 \mathrm{M} \mathrm{NaOH}$ solution for 15 minutes followed by 15 minutes of rinsing in deionized water and placed in an $80^{\circ} \mathrm{C}$ drying oven overnight.

\subsection{Sample types}

Chitosan was supplied by Vanson HaloSource of Seattle Washington. Two grades of chitosan were studied, based on the percentage of chitosan monomer units in the polymer, with the remainder being chitin. Grades were designated as types "A", or "C", having $82.1 \%$ and $93.8 \%$ chitosan polymer units respectively. The chitosan percentage is also referred to as "de-acetylation factor", since the chemical process of making chitosan involves reacting chitin to remove the acetyl functional group [1]. Five samples of each of the two grades of chitosan were spun-cast.

\section{Data analysis}

\subsection{Optical anisotropy and generalized ellipsometry}

In transparent materials where the dielectric permittivity depends on the direction light travels in the material, important optical effects can be observed. Mainly, the speed of light through the material is 
direction dependant. This is known as optical anisotropy. This difference is measured as a difference in the index of refraction,

$$
\Delta n=n_{2}-n_{1},
$$

where $\Delta n$ is commonly referred to as birefringence. There can also be differences in extinction coefficient $k$.

In spin-cast chitosan films there was observed a difference in index of refraction for the in-plane vs. out-of-plane directions, known as uniaxial anisotropy. All three coordinate directions showing a different index is referred to as biaxial anisotropy. Uniaxial anisotropy observed for spin-cast chitosan films is most likely the result of long polymer chains lying within the plane of the sample, due to flow induced during spin-casting.

Generalized ellipsometry (GE) combines the theory and experiment of anisotropy in layered structures [4]. GE enables new insights into physical phenomena of novel thin film materials. The analytical approach for solving the light propagation in complex optical systems is to calculate the Jones matrices for anisotropic homogeneous layered systems [5].

\subsection{Infrared spectroscopic ellipsometry}

Infrared spectra were taken on a commercial infrared variable angle spectroscopic ellipsometer (IRSE) [6-8]. The particular system used was modified to include a custom-made sample holder providing dry air purge gas around the chitosan sample. This prevented water absorption by chitosan during data acquisition.

To successfully optically model chitosan films in the infrared, data were taken on each blank silicon wafer before chitosan was spin-cast. Substrate characterization was required in the infrared because a small difference in dopant concentration of each wafer had a significant effect on the free carrier concentration and thus on optical properties.

IRSE data on each wafer were fit using a Drude model to account for infrared absorption due to free carriers [9]. The Drude model is obtained from the Lorentz oscillator model by setting the restoring force to zero. The Lorentz oscillator model for the dielectric function is given by,

$$
\hat{\varepsilon}(E)=\varepsilon_{1 \infty}+\sum_{n} \frac{A_{n}}{E_{c n}^{2}-E^{2}-i B_{n} E},
$$

where, $\varepsilon_{1 \infty}$ is the real part of the dielectric function as the energy goes to infinity, and $A_{n}, B_{n}$, and $E_{c n}$ are the strength, broadening (loss), and center energy of the $n$th oscillator, respectively. Setting the centering energy to zero, as well as writing $A$ and $B$ in terms of $\rho$, the resistivity of the material and $\tau$, the mean scattering time between collisions of the free carriers, gives the "Rho-Tau" form of the Drude model $[9,10]$.

$$
\hat{\varepsilon}(E)=\varepsilon_{1 \infty}-\frac{\hbar^{2} / \varepsilon_{0}}{\rho \tau\left(E^{2}+\mathrm{i} \hbar E / \tau\right)} .
$$

This relates the resistivity and mean scattering time to the dielectric function and allows any differences in free carrier density from sample to sample to be accounted for. The layer thickness of each chitosan 
spin-cast sample was determined from SE data taken in a dry nitrogen purged ellipsometer, using a Cauchy model fit, as previously described [2,3].

Infrared SE data were modeled using Gaussian oscillators to account for absorptions due to chemical bond vibrations [11-17]. Oscillator center energy, amplitude, and broadening were fit, one parameter at a time for each individual chemical-bond related peak in the data. Once this initial fit scheme was completed, the energy, amplitude, and broadening were simultaneously floated in the regression analysis for each individual oscillator. Upon completion of fitting for each oscillator individually, data were fit for in groups of 2 or 3 oscillators, and finally all three fit parameters were floated for all oscillators simultaneously in a total parameter fit. This step-wise fitting procedure was required to arrive at a best fit, while preventing some oscillators from showing unphysical results such as very small (or large) broadening, or negative amplitudes. Beginning a fit by allowing too many fit parameters to float at once until close to a correct set was known, resulted in a high probability the fit would not work.

\section{Results}

Modeling provides information about oscillator strength and relative abundance of a particular chemical bond per unit volume. Knowing film density and molecular weight of a single chitosan monomer unit, the number of bonds per unit volume can be calculated. Table 1 shows the relative abundance of bonds of interest for type A chitosan, and Table 2 lists the relative abundance for type $\mathrm{C}$ chitosan. Taking the density of spin-cast chitosan to be $1.1 \mathrm{~g} / \mathrm{cm}^{3}$, Table 3 lists the number of bonds per cubic nanometer for both type A and type C chitosan.

\subsection{Bond abundance and oscillator strengths}

Table 3 lists abundance of chemical bonds that should be of interest to a spectroscopist because they are given as an amount per unit volume. This abundance should correspond to the abundance given by Infrared spectroscopic ellipsometry (IRSE). In IRSE absolute dielectric constants are determined, so the abundance can be experimentally derived by examining area under the peaks in $\varepsilon_{2}$, the imaginary part of the dielectric constant. The ratios of these areas should match the ratio of absolute abundance

Table 1

Relative abundance of chemical bonds in type A chitosan

\begin{tabular}{ll}
\hline $\mathrm{C}-\mathrm{O}-\mathrm{C}$ & 1.5 \\
$\mathrm{~N}-\mathrm{H}$ & 1.821 \\
$\mathrm{O}-\mathrm{H}$ & 2 \\
$\mathrm{C}=\mathrm{O}$ & 0.179 \\
\hline
\end{tabular}

Table 2

Relative abundance of chemical bonds in type $\mathrm{C}$ chitosan

\begin{tabular}{ll}
\hline $\mathrm{C}-\mathrm{O}-\mathrm{C}$ & 1.5 \\
$\mathrm{~N}-\mathrm{H}$ & 1.938 \\
$\mathrm{O}-\mathrm{H}$ & 2 \\
$\mathrm{C}=\mathrm{O}$ & 0.062 \\
\hline
\end{tabular}


Table 3

Absolute abundance of chemical bonds per cubic nanometer

\begin{tabular}{cc}
\hline & \# of bonds per $\mathrm{nm}^{3}$ \\
\hline Type A & \\
$\mathrm{C}-\mathrm{O}-\mathrm{C}$ & 5.89 \\
$\mathrm{~N}-\mathrm{H}$ & 7.15 \\
$\mathrm{O}-\mathrm{H}$ & 7.85 \\
$\mathrm{C}=\mathrm{O}$ & 0.70 \\
Type C & \\
$\mathrm{C}-\mathrm{O}-\mathrm{C}$ & 6.07 \\
$\mathrm{~N}-\mathrm{H}$ & 7.84 \\
$\mathrm{O}-\mathrm{H}$ & 8.09 \\
$\mathrm{C}=\mathrm{O}$ & 0.25 \\
\hline
\end{tabular}

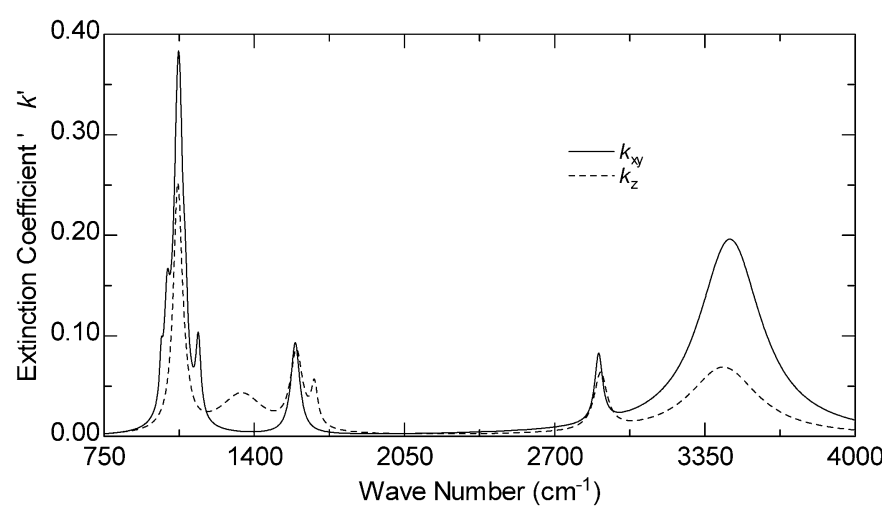

Fig. 2. Extinction coefficient $(k)$ as a function of wavelength for type A chitosan in the infrared.

given in Table 3. Figure 2 and Fig. 3 show the extinction coefficient $(k)$ as a function of wavenumbers in the infrared for type $\mathrm{A}$ and type $\mathrm{C}$, respectively. The imaginary dielectric constant $\varepsilon_{2}$ is related to the extinction coefficient $k$ via,

$$
\varepsilon_{1}=n^{2}-k^{2} \quad \text { and } \quad \varepsilon_{2}=2 n k .
$$

However it is the imaginary dielectric constant which most easily relates to quantum mechanics. The area under a peak in $\varepsilon_{2}$ is directly related to the abundance of a particular chemical bond and its oscillator strength. Working back, oscillator strength can be derived knowing the abundance of the bond and area under the $\varepsilon_{2}$ peak.

Figures 4 and 5 show the index of refraction $(n)$, as a function of wavelength from 750 to $4000 \mathrm{~cm}^{-1}$. Table 4 gives the areas under the $\varepsilon_{2}$ peaks for both type $\mathrm{A}$ and type $\mathrm{C}$ chitosan. The comparison of type A to type $\mathrm{C}$ for each bond type is in good agreement with the calculated abundance for each particular bond. A direct bond to bond comparison can only be performed when oscillator strength is factored into the comparison. Table 5 lists the relative oscillator strength for each of the bond types listed normalized to $\mathrm{O}-\mathrm{H}$. Note that the strongest oscillator is the carbonyl bond which happens to be the only double bond in the biopolymer films. These were calculated by taking area under the peak in $\varepsilon_{2}$ and dividing it by the absolute abundance of each chemical bond and then normalizing to $\mathrm{O}-\mathrm{H}$ dividing by 9.465 , the area 


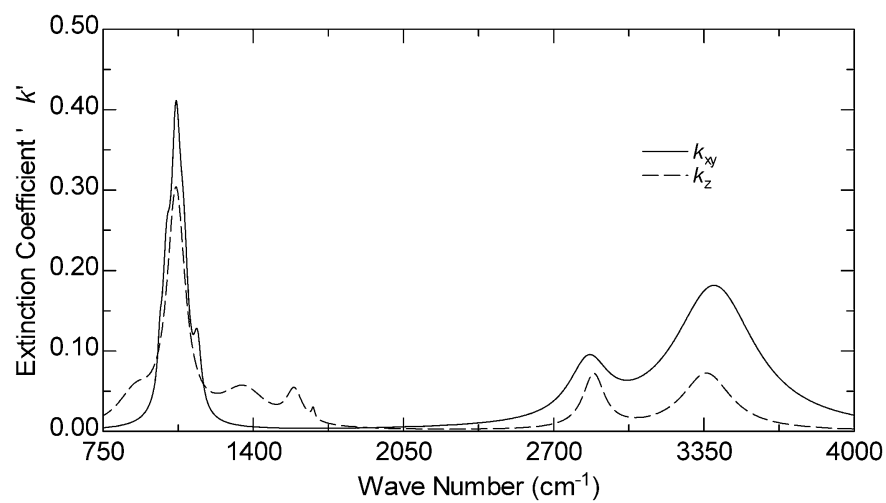

Fig. 3. Extinction coefficient $(k)$ as a function of wavelength for type $\mathrm{C}$ chitosan in the infrared.

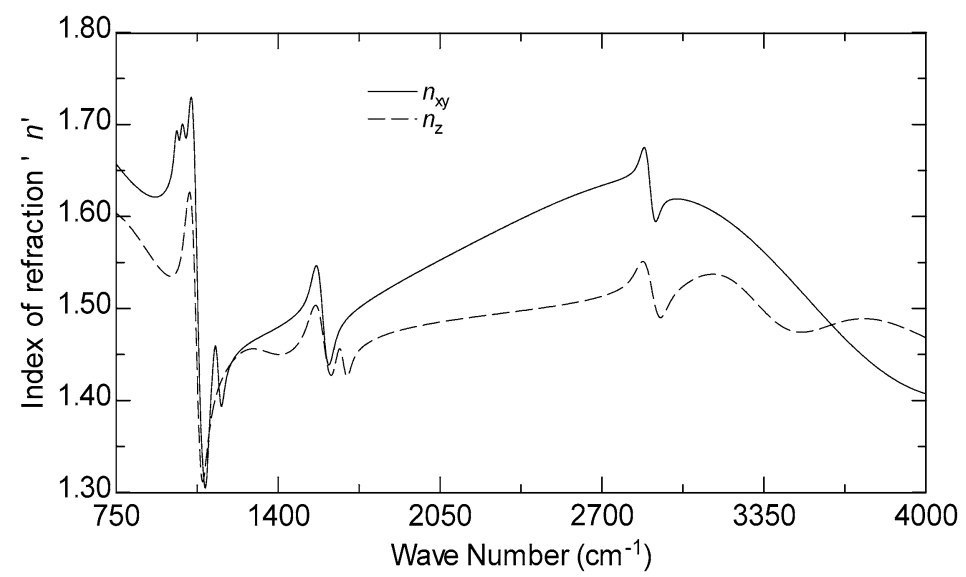

Fig. 4. Index of refraction ( $n$ ) as a function of wavelength for type A chitosan in the infrared.

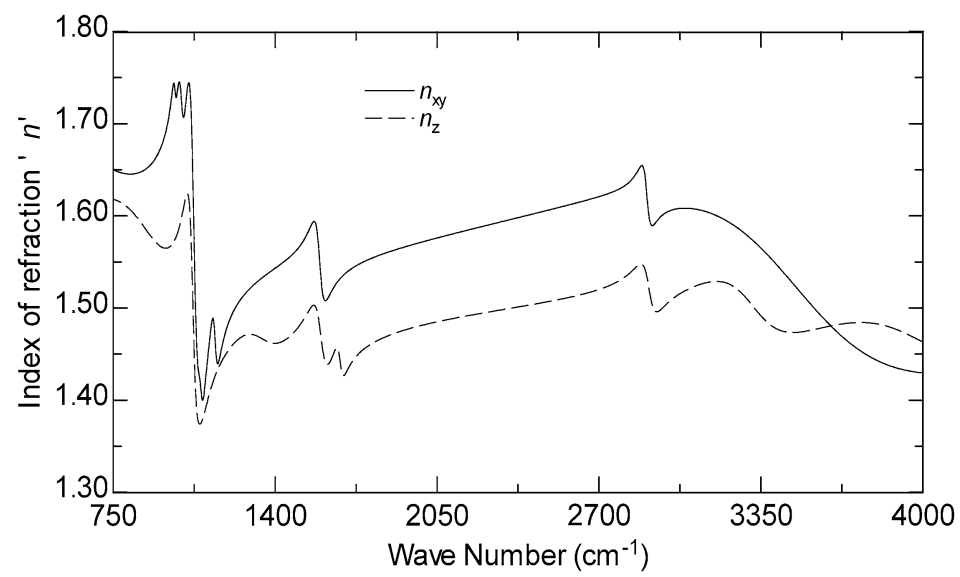

Fig. 5. Index of refraction $(n)$ as a function of wavelength for type $\mathrm{C}$ chitosan in the infrared. 
Table 4

\begin{tabular}{lcc}
\multicolumn{3}{c}{ Areas under $\varepsilon_{2}$ peaks (arbitrary units) } \\
\hline & Type A & Type C \\
\hline $\mathrm{O}-\mathrm{H}$ & 74.3 & 75.6 \\
$\mathrm{~N}-\mathrm{H}$ & 11.6 & 12.2 \\
$\mathrm{C}=\mathrm{O}$ & 14.2 & 6.4 \\
$\mathrm{C}-\mathrm{O}-\mathrm{C}$ & 33.8 & 35.1 \\
\hline
\end{tabular}

Table 5

Relative oscillator strength of bonds in chitosan

\begin{tabular}{lc}
\hline Bond & Strength \\
\hline $\mathrm{O}-\mathrm{H}$ & 1.00 \\
$\mathrm{~N}-\mathrm{H}$ & 0.17 \\
$\mathrm{C}=\mathrm{O}$ & 2.14 \\
$\mathrm{C}-\mathrm{O}-\mathrm{C}$ & 0.61 \\
\hline
\end{tabular}

under the $\mathrm{O}-\mathrm{H}$ peak in arbitrary units. Relative oscillator strength can be used to determine the amount of a particular bond added upon modification.

\subsection{Chemical anisotropy}

Anisotropy is detectable for both types of chitosan used. For simplicity of this paper, only plots of the extinction coefficient are shown. There are two major points found in Figs 2 and 3. First, note that for both chitosan types the $\mathrm{OH}$ stretch and $\mathrm{NH}$ stretch modes have anisotropic character with the in-plane response being greater than the out-of-plane. This is seen as the larger solid line peaks versus the dashed line out-of-plane peaks. This anisotropy indicates a statistically preferred in-plane orientation of the $\mathrm{OH}$ and $\mathrm{NH}$ bonds. The $\mathrm{OH}$ stretch mode is shifted to lower wavenumbers indicating the hydroxyl groups are strongly hydrogen bonded with each other [1].

The $\mathrm{C}-\mathrm{N}-\mathrm{H}$ bending mode at $1660 \mathrm{~cm}^{-1}$ is detectable only for the out-of-plane direction in both Figs 2 and 3. The $\mathrm{C}-\mathrm{O}$ bond at $1160 \mathrm{~cm}^{-1}$ is detectable in-plane only, for both types of chitosan. This corresponds to having the chitosan backbone lie within the plane of the sample due to the spincasting process. This hypothesis is also supported by the anisotropy seen in the $\mathrm{C}-\mathrm{C}$ backbone peak centered at $1050 \mathrm{~cm}^{-1}$. This peak, the tallest in the extinction coefficient plots, shows a preferred inplane orientation. All identifiable IR chemical signatures are in Table 6.

\section{Conclusions}

In this work we determined the infrared dielectric response of spin-cast chitosan films from $750 \mathrm{~cm}^{-1}$ to $4000 \mathrm{~cm}^{-1}$ using spectroscopic ellipsometry. Infrared index of refraction and extinction coefficients were determined using ellipsometric data fits to dispersion models based on Gaussian shaped oscillators. This analysis showed that optical anisotropy was present in the samples over the entire infrared region studied. Analysis of oscillator strengths can be performed when the abundance of chemical bonds is known and the area under each peak of the imaginary part of the dielectric function are determined. This procedure was successfully followed for two types of spin-cast chitosan films studied. 
Table 6

Functional group peak assignments for solid state chitosan

\begin{tabular}{cl}
\hline Position $\left(\mathrm{cm}^{-1}\right)$ & Functional group \\
\hline 3320 & OH stretch \\
2900 & NH stretch \\
1660 & C-N-H bending \\
1600 & C=O \\
1160 & C-O \\
1050 & C-C fingerprint \\
1030 & C-N \\
970 & C-C, C-N fingerprint \\
\hline
\end{tabular}

\section{Acknowledgement}

We wish to acknowledge financial support from the Army Research Laboratory under contract \# 251105-0005-016, as well as the College of Engineering and Technology at the University of NebraskaLincoln.

\section{References}

[1] S. Dumitriu, Polymeric Biomaterials, 2nd edn, Marcel Dekker, Inc., New York, 2002.

[2] W.H. Nosal, D.W. Thompson, L. Yan, S. Sarkar, A. Subramanian and J.A. Woollam, UV-visible-infrared optical and AFM study of spin-cast chitosan films, Colloids and Surfaces B: Biointerfaces 43 (2005), 131-137.

[3] W.H. Nosal, D.W. Thompson, L. Yan, S. Sarkar, A. Subramanian and J.A. Woollam, Infrared optical properties and AFM of spin-cast chitosan films chemically modified with 1,2-epoxy-3-phenoxy-propane, Colloids and Surfaces B: Biointerfaces 43 (2005), in press.

[4] M. Schubert, B. Rheinlander, J.A. Woollam, B. Johs and C.M. Herzinger, Extension of rotating-analyzer ellipsometry to generalized ellipsometry: determination of the dielectric function tensor from uniaxial $\mathrm{TiO}_{2}$, Journal Optical Society of America A 13 (1996), 875-883.

[5] J.F. Elman, J. Greener, C.M. Herzinger and B. Johs, Characterization of biaxially-stretched plastic films by generalized ellipsometry, Thin Solid Films 314 (1998), 814-818.

[6] H.G. Thompkins and W.A. McGahan, Spectroscopic Ellipsometry and Reflectometry: A User's Quide, Wiley, New York, 1999.

[7] J.A. Woollam, B. Johs, C.M. Herzinger, J. Hilfiker, R. Synowicki and C.L. Bungay, Overview of Variable Angle Spectroscopic Ellipsometry (VASE), part I: Basic theory and typical applications, Critical Reviews of Optical Science and Technology CR72 (1999), 3-28.

[8] B. Johs, J.A. Woollam, C.M. Herzinger, J. Hilfiker, R. Synowicki and C.L. Bungay, Overview of Variable Angle Spectroscopic Ellipsometry (VASE), part II: Advanced applications, Critical Reviews of Optical Science and Technology CR72 (1999), 29-58.

[9] T.E. Tiwald, D.W. Thompson, J.A. Woollam, W. Paulson and R. Hance, Application of IR variable angle spectroscopic ellipsometry to the determination of free carrier concentration depth profiles, Thin Solid Films 313 (1998), 661-666.

[10] F. Wooten, Optical Properties of Solids, Academic Press, New York, 1972.

[11] D. Naumann, Applied Spectroscopy Reviews 36 (2001), 239-298.

[12] B. Stuart and D.J. Ando, Biological Applications of Infrared Spectroscopy, Published on behalf of ACOL (University of Greenwich) by John Wiley, Chichester, New York, 1997.

[13] M. Diem, Introduction to Modern Vibrational Spectroscopy, Wiley, New York, 1993.

[14] R. Garrett and C.M. Grisham, Biochemistry, 2nd edn, Saunders College Pub., Fort Worth, 1999.

[15] H.H. Mantsch and D. Chapman, eds, Infrared Spectroscopy of Biomolecules, 1996.

[16] J. McMurry, Organic Chemistry, 3rd edn, Belmont, Cole, Brooks, CA, 1992.

[17] R.A. Alberty and R.J. Silbey, Physical Chemistry, Wiley, New York, 1992. 


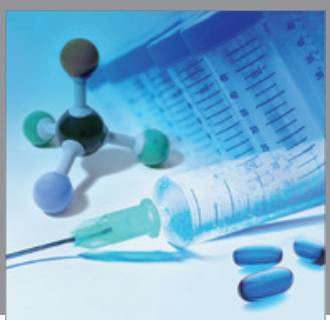

International Journal of

Medicinal Chemistry

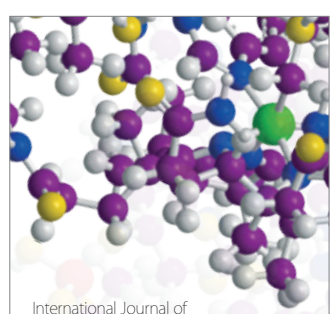

Carbohydrate Chemistry

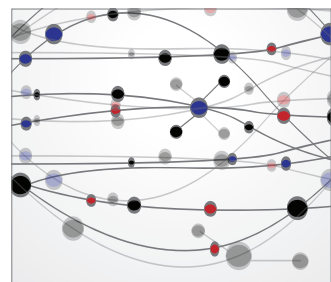

The Scientific World Journal
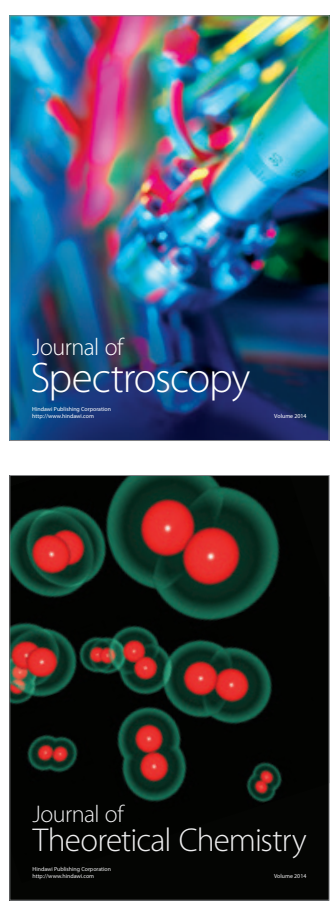
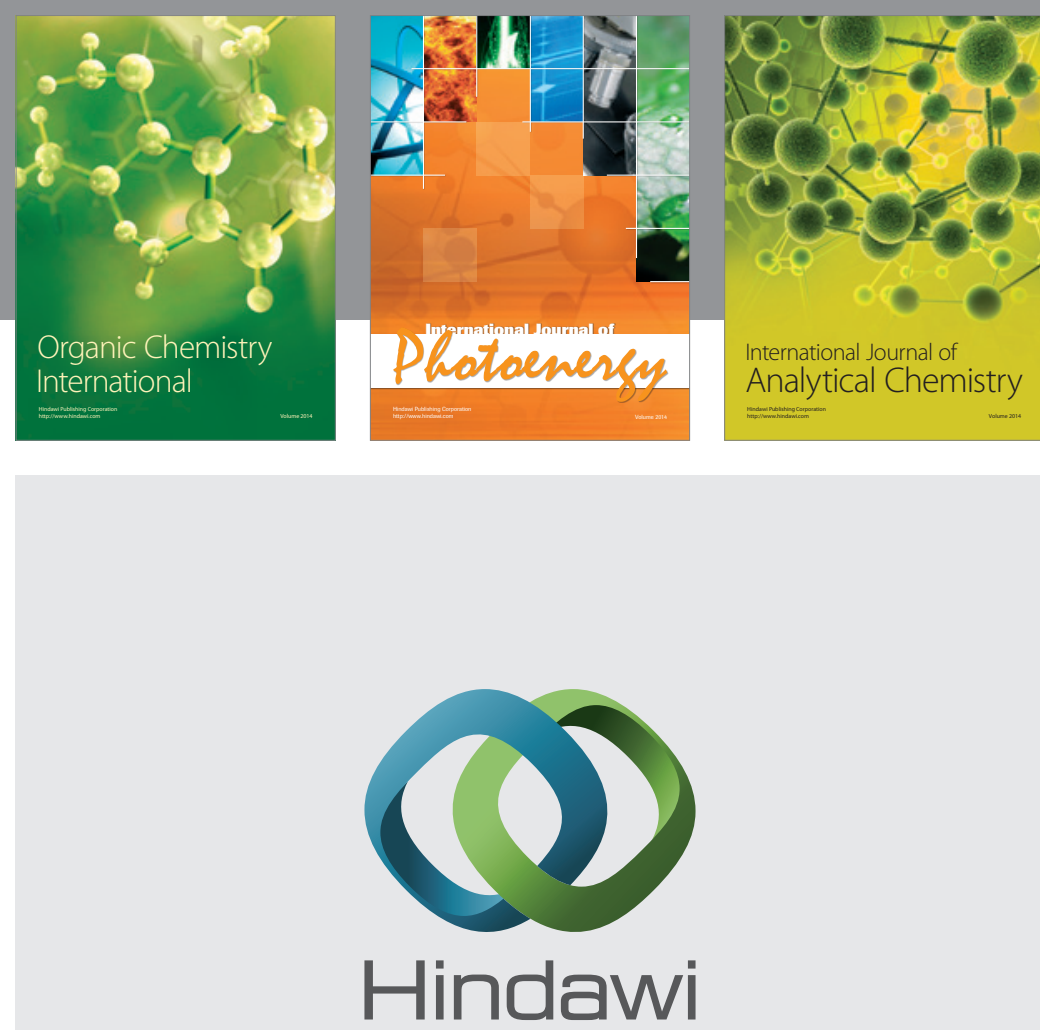

Submit your manuscripts at

http://www.hindawi.com
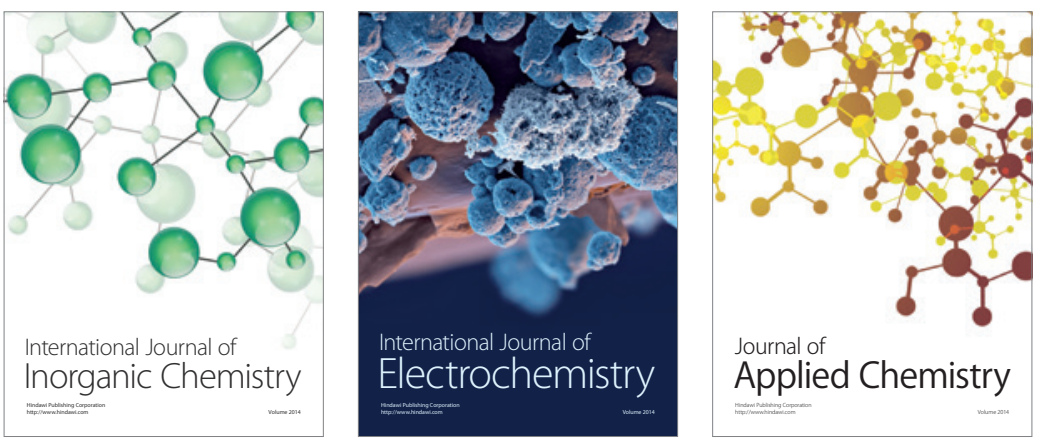

Journal of

Applied Chemistry
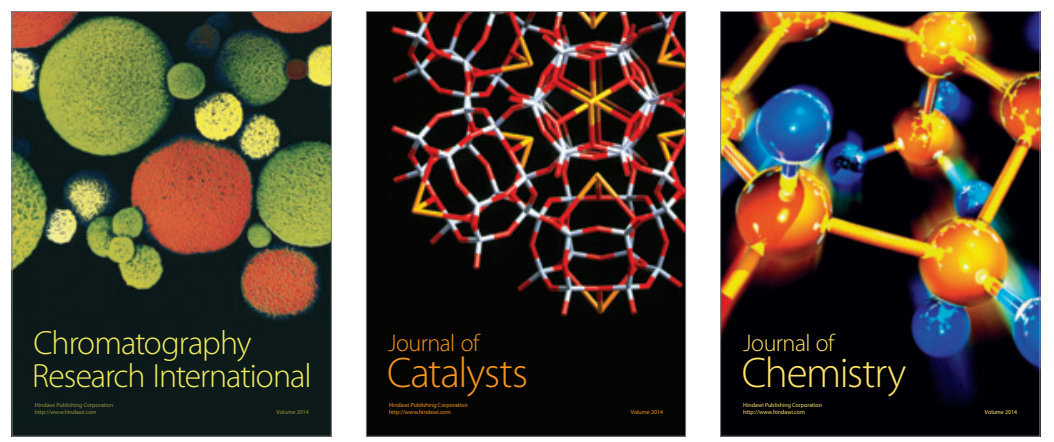
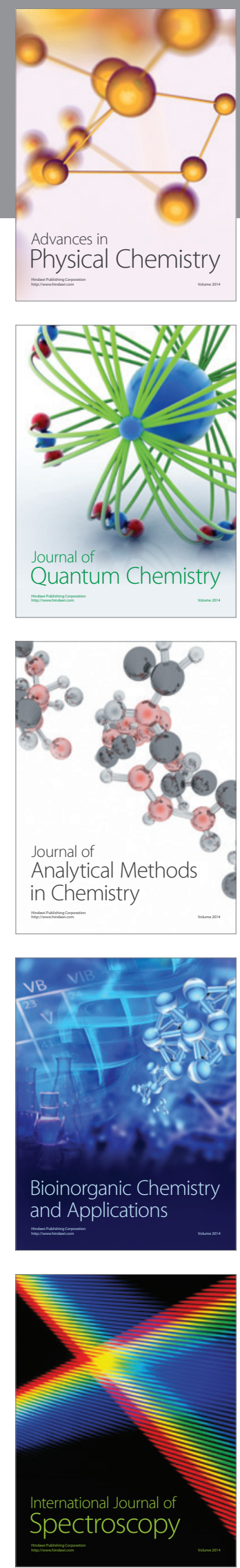